\title{
Numerical investigation of the performance of Heller type cooling towers in different arrangements from the perspective of air intake flow rate
}

\author{
H. Reshadatjoo, S. Yekani Moltagh ${ }^{\mathrm{a}}$ and I. Mirzayi \\ Mechanical Engineering of Urmia University of Technology, 0098 Urmai, Iran
}

Received 18 October 2014, Accepted 5 January 2015

\begin{abstract}
In this article, Heller type cooling towers have been simulated in four different arrangements under the free convection and cross wind conditions. The obtained results from the numerical work have been compared with the available experimental results. By studying the flow pattern, the influential factors on the reduction of air intake flow rate (AIFR) resulting from the interaction of the cooling towers, and also the negative effects of cross wind in different tower arrangements have been investigated.
\end{abstract}

Key words: Heller type cooling tower / air intake flow rate / cooling tower arrangement

\section{Introduction}

The issue of the cooling towers' performance being affected by environmental factors, including wind, has grabbed the attention of researchers for many years. A different viewpoint of this issue, and the progresses made in computer aided simulation and wind tunnel testing has attracted even more attention in recent years. Furthermore, the way the cooling towers operate under cross wind conditions is a research subject that recently has come to the forefront.

Many investigations have been carried out on the problem of efficiency loss in the dry cooling towers under cross wind conditions, but the main cause of this efficiency drop has not been exactly determined. The following is a brief account of the research works done on the subject.

In 1988, Radosavljevic and Spalding [1], simulated the fluid flow and temperature distribution in a wet cooling tower affected by cross wind. In 1993, Bergstrom et al. [2] performed a two-dimensional wind flow modeling on a tower.

In the same year, Preez and Kroger [3] studied the wind effect on a natural draft dry cooling tower. They used the simplest turbulence model based on the eddyviscosity to study the flows inside and on the periphery of the tower. In addition, they introduced the windbreak walls as a tool for minimizing the cross wind effect on the thermal performance of towers.

${ }^{\text {a }}$ Corresponding author: syekani@iust.ac.ir
In 1995, Wei et al. [4] used complete sample models and wind tunnel testing to show the adverse effects of wind on the functioning of dry cooling towers. They utilized a system of hot water circulation and radiators to simulate the thermodynamic process of dry cooling towers. The dimensionless parameter of wind effect coefficient, CW (for describing the wind effect on the efficiency of tower radiators), was defined and measured by them. Their experimental results demonstrated that improper pressure distribution at the tower inlet and also, the elimination of plume at the top portion of tower are the factors responsible for efficiency loss in the tower.

In 1996, Derksen et al. [5], in a continuous research on a cooling tower, investigated the effects of wind on the air intake flow rate. These studies were prompted by problems encountered in the operation of cooling towers in the west of Canada. They conducted their work through wind tunnel tests on a $1 / 25$ scale model of a wet cooling tower. They studied the external flow patterns, pressure characteristics, and the air intake flow rate, and finally discovered that the wind-facing side of the tower has an almost $45 \%$ flow rate increase and the opposite rear side has a $19 \%$ flow rate decrease. This flow imbalance was the cause of ice formation on the inlet side. Derksen and colleagues later showed that using simple walls at the upstream of flow can be beneficial in establishing equilibrium between the intake flow rates of the two sides of towers. These walls exert a drag force on the velocity field, which causes a loss of wind momentum. In continuation, they used numerical simulations to estimate the amount 
of heat transfer. They applied the two-dimensional finite volume method to model the flow on the tower.

In 1999, Su et al. [6], using the NSTR software, modeled the temperature and air flow distribution in a Heller cooling tower under the cross wind effect. They applied the standard $k-\varepsilon$ model as the turbulence model and investigated two cases: natural convection without the cross wind, and forced convection at wind speeds of 5 and $10 \mathrm{~m} . \mathrm{s}^{-1}$. Although, they identified several factors for the efficiency loss, they were not successful in suggesting a solution for reducing the effect of wind on tower.

In 2001, Bornoff et al. [7] numerically studied the encounter of the plumes of two cooling towers exposed to wind. Their results showed that in a tandem arrangement, the encounter between the plumes of the two towers is due to the influence of counter-rotating pair of vortices inside the plumes. The modeled towers were of the forced type and their simulation depicted two cylinders with flow injected from the bottom of towers at a fixed rate.

In 2002 and 2003, Kapas [8] first studied the flow characteristics of Heller type cooling towers at different cooling delta angles, and then, investigated the overall flow behavior of the towers affected by wind. In the first study, by using the "Fluent" software and applying 2-D models, he evaluated 4 delta angles, and for each case, calculated the pressure drop of the flow. In his research work, Kapas applied a specific value for the rate of flow passing over the deltas, which is not a valid assumption for natural draft towers; basically, the rate of flow passing through the deltas depends on the pressure loss.

In 2004, Al-Waked and Behnia [9], by using the "Fluent" software, did a 3-D modeling of a tower and simulated the internal and external flows. They used the $k-\varepsilon$ turbulence model and, by introducing a new parameter, stipulated that the wind speed profile is an important factor in correctly simulating the flow around the tower. They also suggested the windbreak walls as a solution for reducing the adverse effects of wind. The type of tower investigated by them was the Hamoun type with the horizontal arrangement of radiators. Another noteworthy point in the work of Al-Waked and Behnia was the use of inlet velocity and outlet pressure boundary conditions. In 2006, Mokhtarzadeh Dehghan et al. [10] numerically investigated the interaction of two turbulent plumes that were issuing from two cylinders next to each other.

Also, in 2006, Zhai and $\mathrm{Fu}$ [11] performed a numerical and experimental study of the effects of two windbreak side walls on the efficiency of a dry cooling tower.

In 2008, Williamsona et al. [12] investigated the effect of radial thermo-fluid fields in a natural draft wet cooling tower.

In the present work, the effect of wind on the functioning of a cooling tower with different arrangements has been considered from a fluid mechanics point of view. Through this study, the phenomena influencing tower performance with regard to air intake flow rate (AIFR), in free and forced convection conditions, have been identified.
Table 1. Specifications of the cooling tower of Shahid Rajaee power plant.

\begin{tabular}{cc}
\hline Tower type & Heller \\
Tower height & $150 \mathrm{~m}$ \\
Tower base diameter & $118.2 \mathrm{~m}$ \\
Tower tip diameter & $64 \mathrm{~m}$ \\
Water pump flow rate & $12600 \mathrm{~m}^{3} / \mathrm{Hr}$ \\
Number of cooling radiators/height & $144 / 15 \mathrm{~m}$ \\
\hline
\end{tabular}

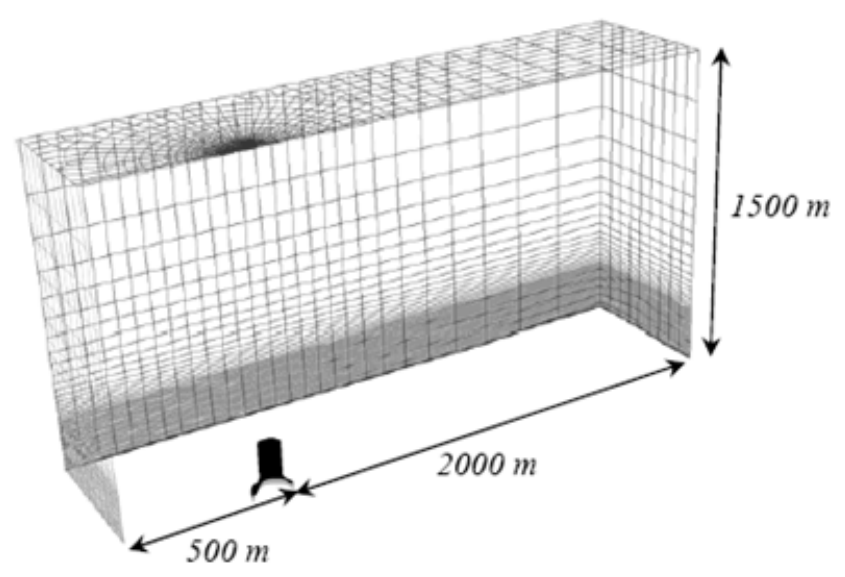

Fig. 1. Dimensions of the solution domain.

\section{Three-dimensional modeling of the indirect dry cooling tower}

\subsection{Geometry and boundary conditions}

In the present research, a tower with similar specifications to those of the dry cooling tower of "Shahid Rajaee" power plant located in central Iran has been studied. Table 1 lists the specifications of the cooling tower of this power plant.

Dimensions of the solution domain, boundary conditions, and the mesh have been presented in Figures 1 and 2, respectively.

Boundary conditions include the velocity inlet, symmetric, wall, and the outlet. The inlet and outlet boundaries have been selected as follows.

\section{Velocity profile of inflowing wind}

An important wind characteristic is its change of velocity with height. According to the work by Al-Waked and Behnia [9], an accurate determination of wind profile plays a significant role in the correct modeling of wind flow around a cooling tower. The relation between the wind velocity and height can be written as:

$$
V(z)=V_{g}\left(\frac{Z}{\delta}\right)^{\alpha}
$$

where $\delta$ is the boundary layer thickness of earth's atmosphere, $V(z)$ is the wind velocity at height $Z, V_{g}$ is the 


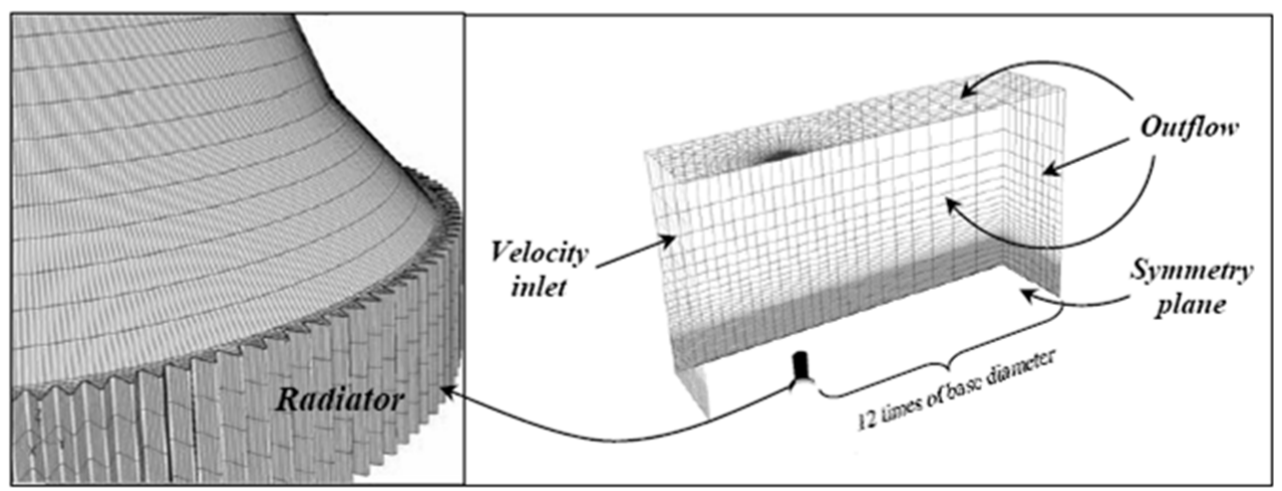

Fig. 2. Boundary conditions and mesh configuration of the model for one cooling tower.

wind velocity at height $\delta$, and $\alpha$ is a number dependent on ground roughness. What is meant by roughness is the unevenness of the ground around the tower, including buildings, trees, hills, etc. In suburban areas, the values of $\delta$ and $\alpha$ are taken as $122 \mathrm{~m}$ and 902, respectively, and those numbers have been used in the present work.

\section{Outlet boundary}

If the outlet boundaries are situated close to solid barriers, then the flow may not become fully developed and may cause large errors. In these situations, the assumption of zero gradient condition cannot be used. It is necessary to place the outlet boundary at a distance of more than 10 times the height of the existing barrier before the downstream flow in order to get accurate results. For the present work, the computational domain has been taken to be almost 12 times the tower height, so that the outlet boundary condition can be safely assumed.

\section{Tower arrangements}

In the present research work, the performance of Heller type cooling towers in four different arrangements has been investigated: arrangement (1) two parallel towers perpendicular to the wind blowing direction (Fig. 3a), arrangement (2) two consecutive towers along the wind direction (Fig. 3b), arrangement (3) three towers on the vertices of an equilateral triangle (Fig. 3c), and arrangement (4) four towers (Fig. 3d). In all the cases, the distance between the towers is twice the diameter of the tower base. Arrangements (1) and (2) are identical in free convection condition.

\subsection{Numerical solution of governing equations}

The governing equations comprise the time-averaged three-dimensional momentum (Navier-Stokes) equations, the energy and continuity equations, and the equations of the turbulence model. In this paper, the open source CFD package, OpenFOAM (Open source Field Operation
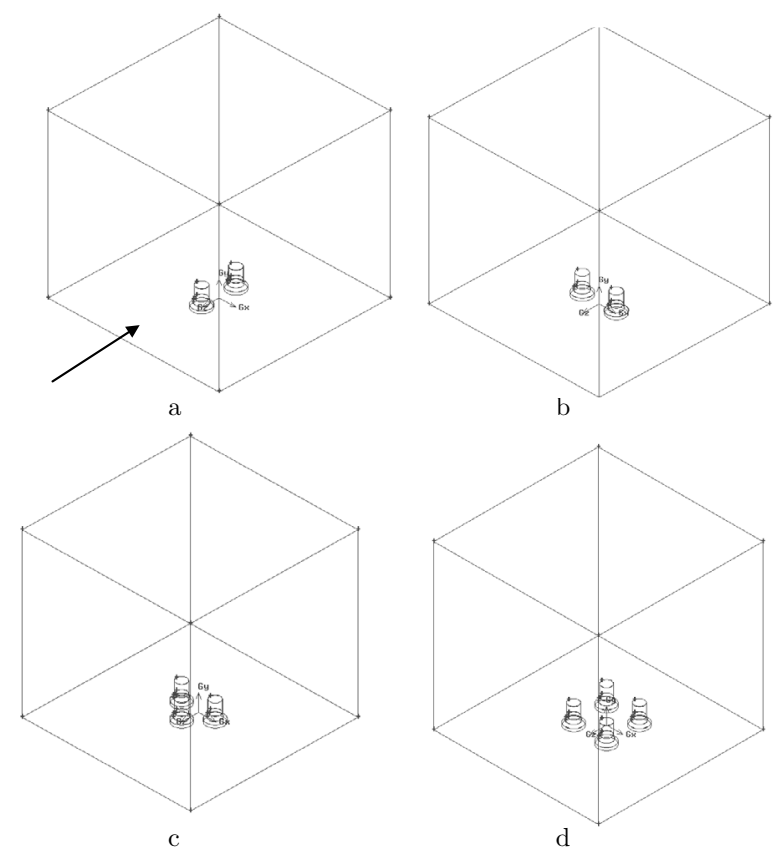

Fig. 3. Cooling towers arrangements.

and Manipulation), which is based on the work of Weller et al. [13], is used for flow simulation. The discretization of the flow governing equations in the OpenFOAM is based on the finite volume method formulated with collocated variable arrangement, with pressure and velocity solved by segregated methods. The OpenFOAM is a $\mathrm{C}++$ code library of classes for writing CFD codes, which includes a well-tested and validated RANS (Reynolds Averaged Navier Stokes) capability. In this study, the $k-\omega$ model is used for turbulence modeling. Also, the standard wall function based on the proposal by Launder and Spalding [14] is used. It has been used most widely for industrial flows. Several numerical convection differencing schemes are available in the OpenFOAM, including the upwind, central difference, QUICK, and the flux limiter schemes. In this work, the limited linear differencing scheme (limited Linear V) [15] is used. It is a bounded high-order scheme that takes into account the direction 


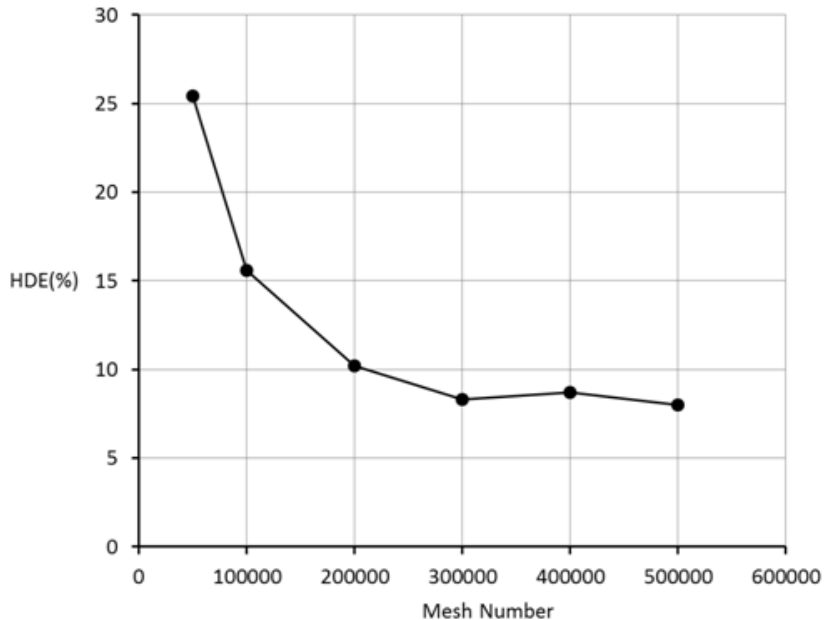

Fig. 4. HDE value versus mesh number for the $10 \mathrm{~m} \cdot \mathrm{s}^{-1}$ wind speed.

of the flow field. For the first time derivative, the choice is the Euler implicit first order. The set of discretized equations was solved by the algebraic multi-grid (AMG) solver for pressure, and by the incomplete-Cholesky preconditioned biconjugate gradient (BICCG) solver for the rest of the variables.

\section{Mesh study and comparison with experimental results}

The only experimental results available from "Shahid Rajaee" power plant are the amount of heat dissipated by the tower at different wind speeds. So, to study mesh independency, the heat dissipation error (HDE) has been defined by Equation (1).

$$
\mathrm{HDE}(\%)=\frac{\left|\mathrm{HD}_{\mathrm{Num} .}-\mathrm{HD}_{\mathrm{Exp} .}\right|}{\mathrm{HD}_{\mathrm{Exp}}} \times 100
$$

The maximum wind speed for which experimental results were available is $10 \mathrm{~m} . \mathrm{s}^{-1}$. Thus, the HDE parameter values have been obtained for various mesh counts at this speed. Figure 4 demonstrates the results related to HDE value versus mesh number.

As the figure shows, at this speed, the results have become almost mesh-independent at mesh number 300000 .

The simulations have taken 14-20 days to perform the computations. To verify the numerical solutions, the results pertaining to HDE at wind speeds of $3,5,8$, and $10 \mathrm{~m} . \mathrm{s}^{-1}$, for which the experimental results are available, have been presented in Figure 5 .

As can be seen, the numerical computations, in comparison with the experimental results, have acceptable error.

To correct mesh selection for the different types of towers arrangements modeling, in windy condition, in Figure 6 , the value of air intake flow rate variation

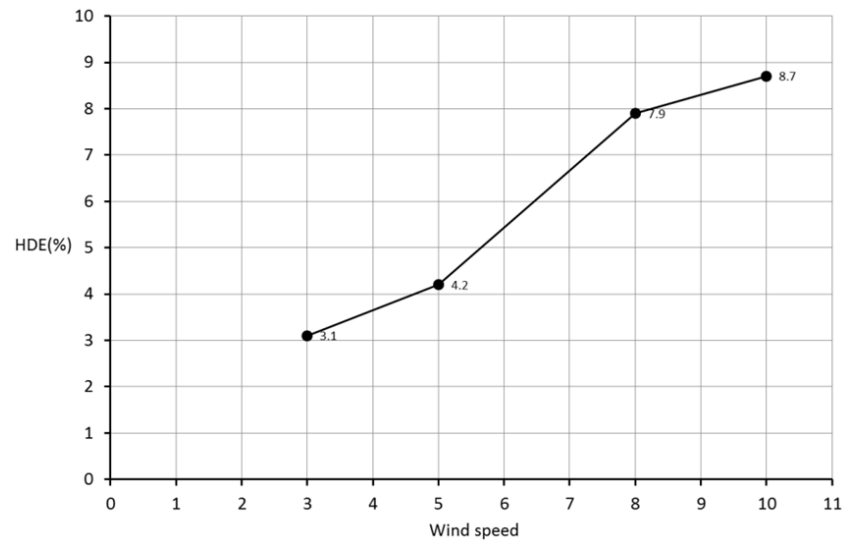

Fig. 5. HDE value versus wind speed.

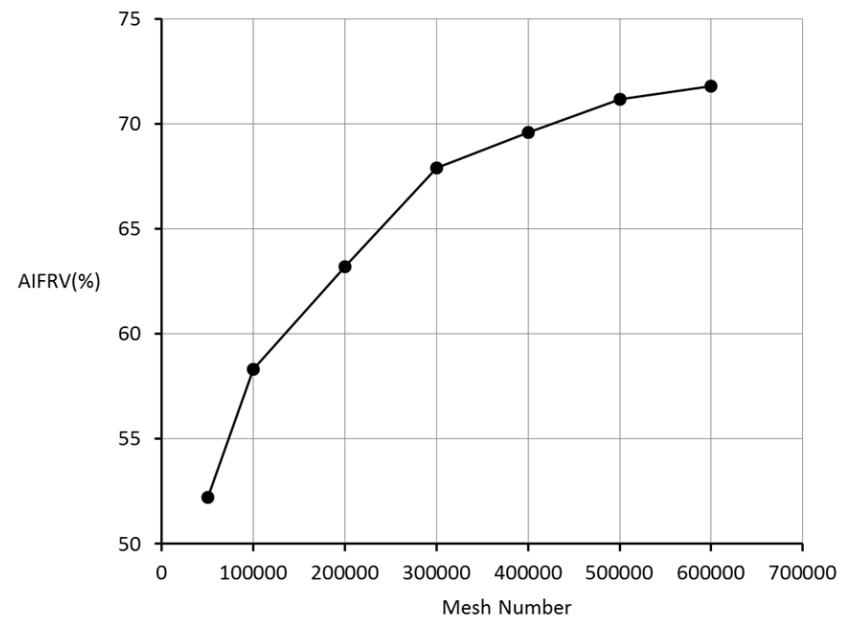

Fig. 6. AIFRV value versus mesh number for the type 4 cooling tower arrangement (wind velocity, $8 \mathrm{~m} . \mathrm{s}^{-1}$ ).

(AIFRV), which is defined as Equation (2), has been presented versus the mesh number, for the wind speed under study (i.e. $8 \mathrm{~m} . \mathrm{s}^{-1}$ ) and type 4 arrangement.

$$
\operatorname{AIFRV}(\%)=\frac{\left|\mathrm{AIFR}_{\text {severalTower }}-\mathrm{AIFR}_{\text {oneTower }}\right|}{\mathrm{AIFR}_{\text {oneTower }}} \times 100
$$

It can be observed that the AIFRV becomes meshindependent at the mesh number 500000 . Therefore, the mesh number 500000 has been used for all the simulations.

\section{Simulation results of free convection flows}

Figure 7 shows the results of velocity contours and velocity vectors related to free convection flows for one tower (Figs. 7a and 7b) and two towers located side by side (Figs. 7c and 7d).

In view of the results, the flow patterns inside and outside the towers are influenced by one another. The plumes exiting the towers interact and are drawn towards each other. Inside the tower, flow symmetry is disturbed 


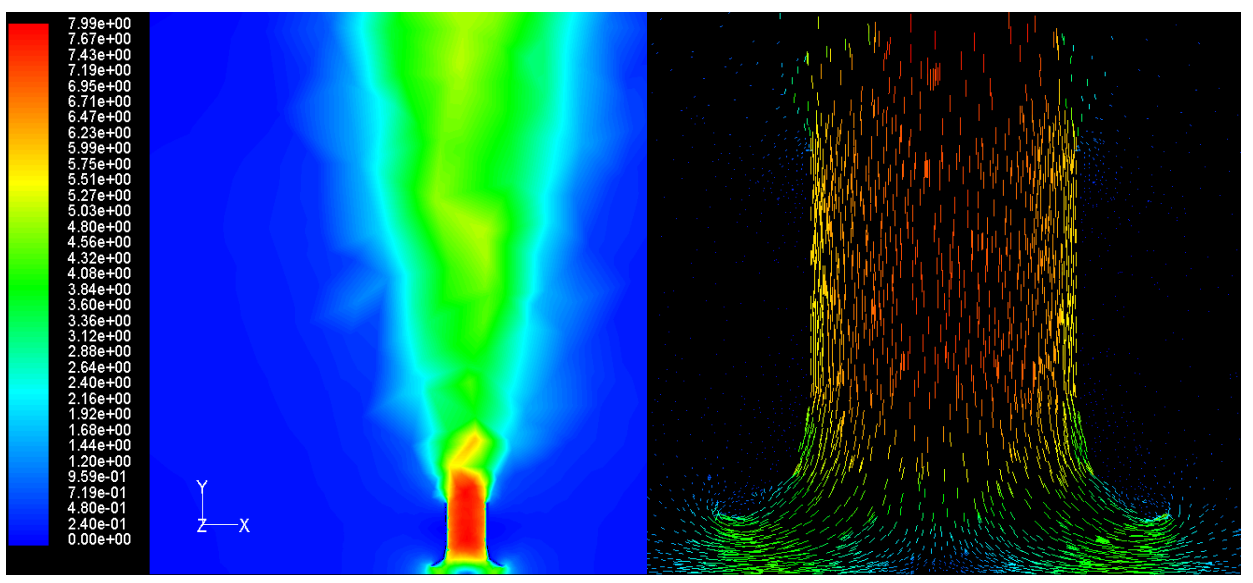

a

$\mathrm{b}$

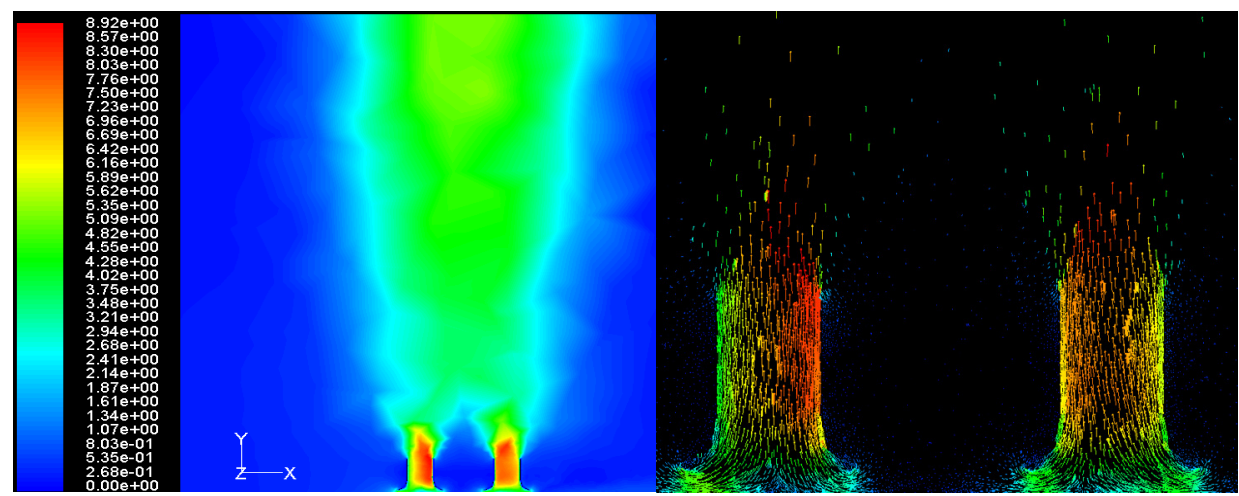

C

d

Fig. 7. Velocity field in free convection conditions: (a) velocity contour (single tower), (b) velocity vectors (single tower), (c) velocity contour (two towers), and (d) velocity vectors (two towers).

Table 2. Comparison of air intake flow rates in free convection condition for different tower arrangements.

\begin{tabular}{ccc}
\hline Arrangement type & AIFR $\left(\mathrm{kg} \cdot \mathrm{s}^{-1}\right)$ & AIFRV (\%) \\
\hline Single tower & 24723.3 & - \\
2 and 1 & 24352.1 & -1.50 \\
3 & 24211.4 & -2.07 \\
4 & 24108.1 & -2.48 \\
\hline
\end{tabular}

and the high velocity region, which in the single tower case is in the central section, shifts towards the tower wall that is close to the second tower. The tower interactions and flow interferences will cause an energy loss and a reduction of flow momentum, and thus, will reduce the rate of air flow passing through the towers.

In Table 2, the results associated with the AIFR and AIFRV for different tower arrangements have been presented.

It is observed that as the number of towers increases, the AIFR is further reduced. In addition, the AIFRV results indicate that in the type 1 and 2 arrangements, a $1.5 \%$ reduction occurs in the rate of air flow into the towers. While, in the type 3 arrangement, a $0.75 \%$ more flow rate reduction is witnessed, relative to the type
2 arrangement. Also, in the type 4 arrangement, a $0.41 \%$ more flow rate reduction, compared to the type 3 , can be seen. This trend shows that as the towers increase in number, the ill effects of the operational interference of towers also increase, and the air intake flow rate is further reduced.

\section{Results of simulation of forced convection}

In this section, the tower's flow pattern and air intake flow rate under the effect of cross wind have been reviewed and the phenomena that are formed inside the tower due to blowing wind have been investigated.

\subsection{Wind effect on the flow inside the tower}

In Figure 8, the stream lines inside the cooling tower have been plotted. As the wind blows, the flow velocity increases in the vicinity of the tower and the pressure drops; thus, in these regions, the air intake of the tower severely declines. Some of the radiators facing the wind, experience a pressure increase in their inlet section that 


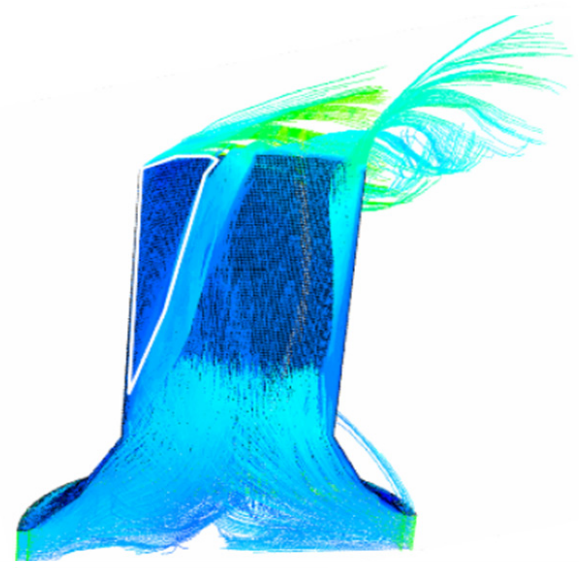

Fig. 8. Vertical flow and the choked zone inside the tower.

causes more air to enter the tower from these regions. On the other hand, some radiators behind the tower are located in the flow separation zone, where the natural tower air intake is not altered very much. But this imbalance in the air intake flow rate from the front and rear of the tower causes the emergence of special phenomena inside the tower which can influence the air intake rate of the tower.

In the lower section inside the tower, just after the radiators, the air intake flow symmetry is disturbed and the static high pressure region inside the tower deviates towards the separation zone. This deviation affects the updraft inside the tower and causes the flow to swirl and rotate. Swirling of the flow wastes the flow energy and is supposed to reduce air intake into the tower.

Another important issue in the investigation of wind effect on flow behavior is the circulation flow that forms inside the tower. On the side where the radiators are facing the wind, the rate of air intake into the cooling tower increases and the flow has a higher speed. When the radial flow moving over the radiators towards the tower center deviates towards the tower top, because of its large momentum, it goes through flow separation during the upward rotational movement. The swirling and rotational flow that forms in these areas causes the air to become entrapped in these regions; as a result, the pressure of air flow inside the tower drops. In addition, this leads to the reduction of a portion of the effective volume inside the tower. Also, the asymmetry of the boundary layer causes the outside wind flow to infiltrate into the tower and choke a large portion of its interior. Part of this choked flow inside the tower has been shown in Figure 8.

Figure 9 illustrates the three-dimensional contour of the flow velocity at the tower tip, for the cases of blowing wind, and choked flow.

As can be seen, in part of the tower tip cross section, the exit flow velocity is close to zero and in another part, the velocity has increased. This shows that the effective cross section of the tower tip is reduced because of flow choking and outside air infiltration into the tower.

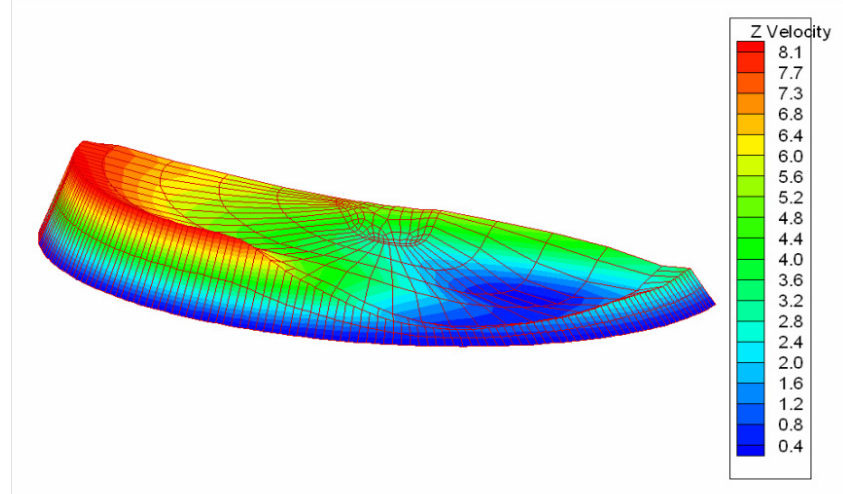

Fig. 9. Three-dimensional velocity contour at the tower tip in choked flow condition.

Therefore, the outflow velocity increases, relative to the free convection case, and its pressure drops.

Another event that occurs at the tower tip is that the air flow, after moving from the base area and upward through the tower's constant level regions, reaches the tower tip and here, by encountering the wind flowing over the tower, runs off course. This phenomenon is also one of the reasons for the tower draft's pressure loss.

To completely delineate the flow situation at the tower tip, in Figures 10 and 11, the diagrams of pressure and velocity distributions along the wind, from $x=-100$ to $x=+100$ and at an elevation of $150 \mathrm{~m}$ (the exact elevation of tower tip) have been given, respectively.

Figure 10 shows that the pressure along the wind increases, the closer one gets to the tower tip, which is due to flow stagnation over the front edge of the mouth. Just after the stagnation point, which is located at $x=-30$, the pressure suddenly drops. This event shows that the tower's internal pressure, because of the presence of warm air flow inside, is less than the stagnation point. Also, it can be observed that the highest static pressure inside the tower, is even less than the static pressure of the wind flow far away. Thus, when the outside wind current encounters the outflow plume of the tower, the potential exists for the air around the tower to infiltrate inside. As one moves ahead on the tower tip area, the static pressure gradually decreases, but again a sudden drop is encountered in the pressure inside the tower. The reason for this is that the out flowing plume has a smaller area from which to exit; therefore, its velocity increases and its pressure drops. In Figure 11 that shows the velocity distribution over the tower tip area, the velocity increase at the outlet edge is quite obvious. In a way, all the facts cited for pressure distribution cases, could be expressed for velocity distribution cases too.

In general, it is observed that the blowing wind disturbs the regular distribution of outflow velocities at the tower mouth, and this disturbance, at some locations, consists of zero vertical velocities or even negative velocities. The locations where the vertical flow velocities have zero or negative values are designated as the tower's choking zones. 


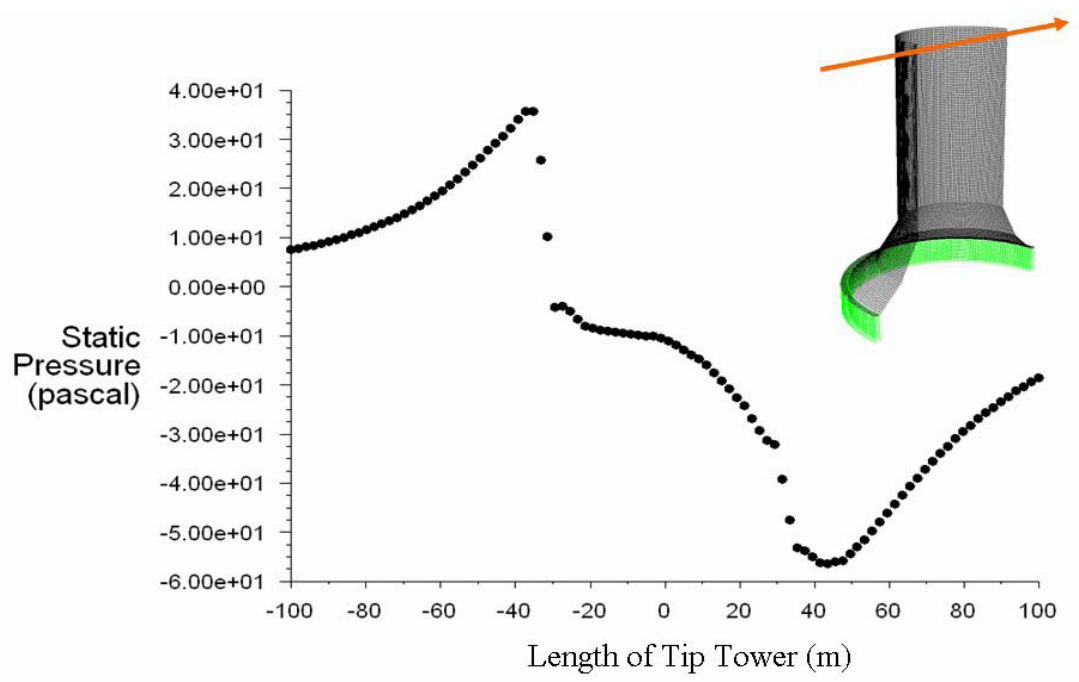

Fig. 10. Diagram of pressure distribution at the tower tip.

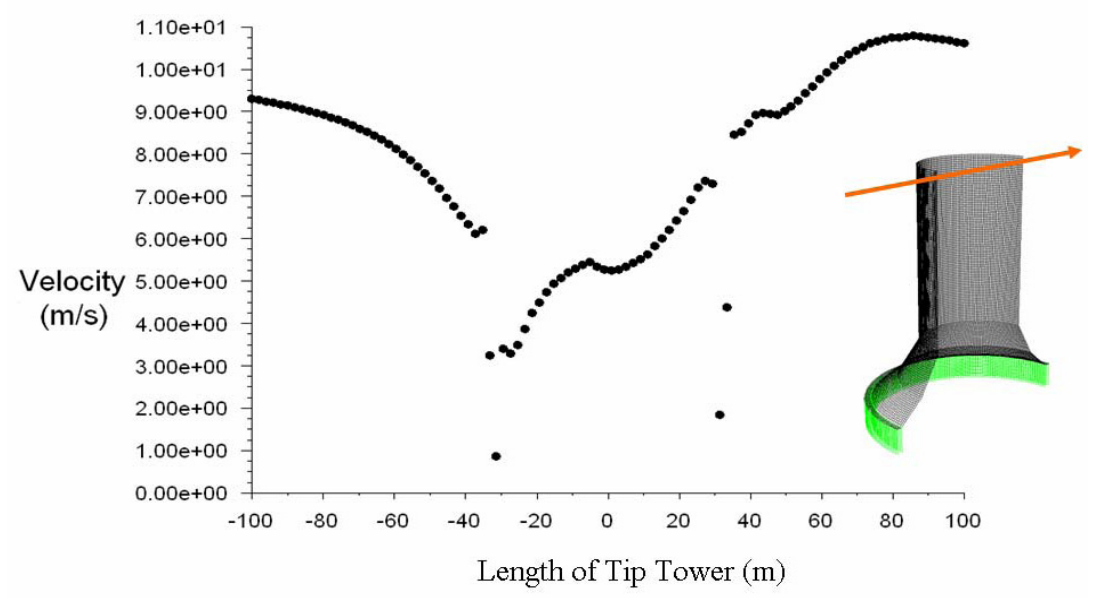

Fig. 11. Diagram of velocity distribution at the tower tip.

The mentioned phenomena are interrelated and cannot be separated; however, the efficiency loss of the tower can be associated with four factors: first, the increase of flow velocity around the side radiators and the reduction of pressure difference between the inside and outside of tower in these regions; second, the deviation caused by the out flowing plume encountering the outside wind flow; three, the wind flow infiltration into the tower through the mouth and the choking of the flow inside and the last, secondary flows and vortical structures.

\subsection{Investigation of the wind effect on AIFR}

In Table 3, the results related to AIFR at various wind speeds have been presented for the single tower case.

It can be observed that with the increase of the wind speed, its adverse effects on AIFR increase. Because, the wind speed increase causes more flow instability and larger vortical, and chocked regions, and it strengthens the factors responsible for AIFR reduction.
Table 3. Comparison of AIFRs at various wind speeds.

\begin{tabular}{cc}
\hline wind speed $\left(\mathrm{m} . \mathrm{s}^{-1}\right)$ & Air intake flow rate $\left(\mathrm{kg} . \mathrm{s}^{-1}\right)$ \\
\hline 0 & 24723 \\
3 & 17219 \\
5 & 11972 \\
8 & 8765 \\
\hline
\end{tabular}

Furthermore, by comparing the results of Tables 2 and 3 , it can be stated that, because wind strongly influences the flow patterns of the towers, it has a more negative effect as compared to the effect of closeness of the cooling towers to one another.

\subsection{Interaction effects of multi towers on AIFR in cross wind conditions}

In Table 4, the effect of wind on the AIFR of several towers, in different arrangements, has been presented. Since the conditions of the front cooling towers that are 


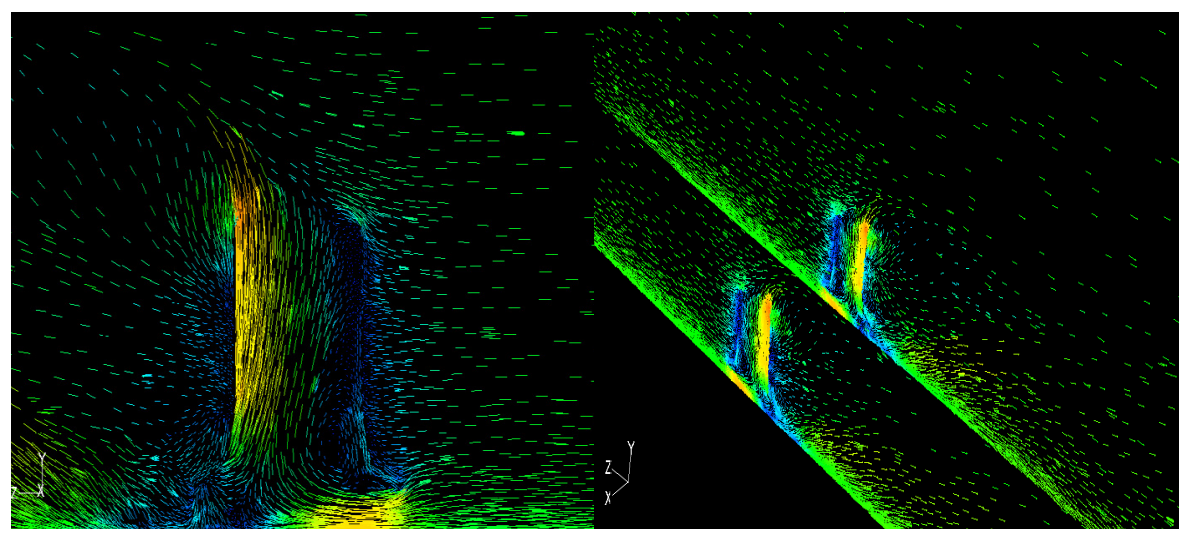

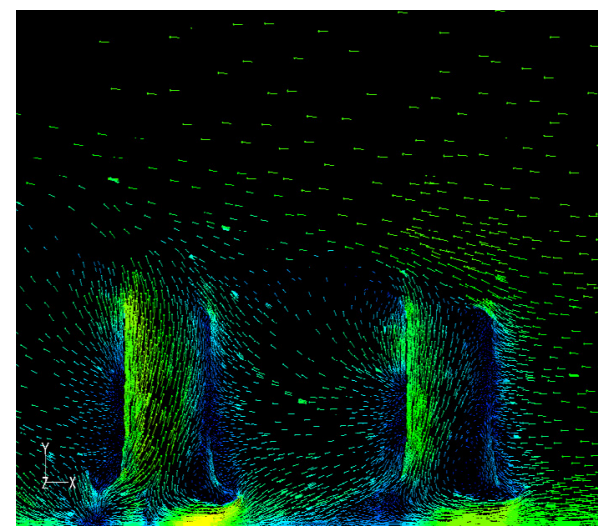

C $\mathrm{b}$

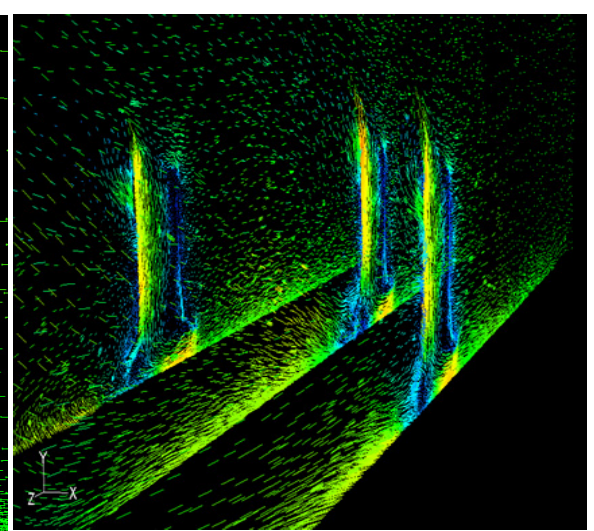

d

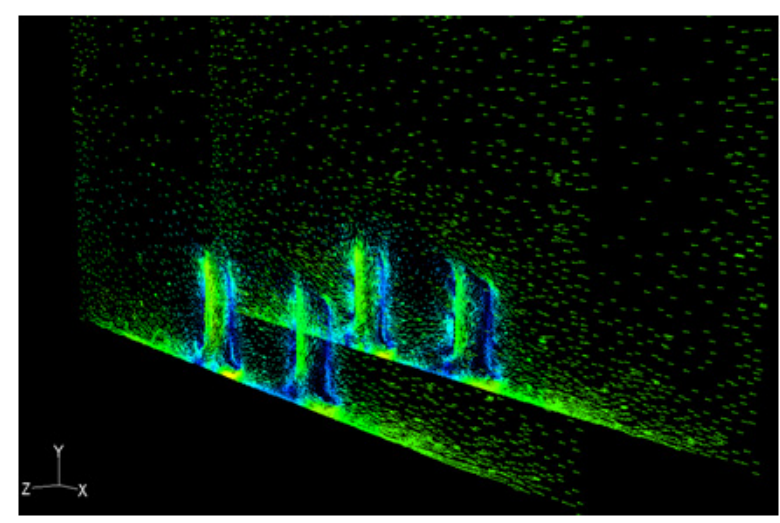

e

Fig. 12. Velocity vectors in different tower arrangements (arrow indicates velocity direction): (a) single tower, (b) type 1, (c) type 2, (d) type 3 , and (e) type 4.

directly exposed to the wind are different from the back towers, the results pertaining to the front towers (on the first line) and back towers (on the second line) have been presented separately.

For the purpose of studying the influential factors on the AIFR, in Figure 12, the flow patterns resulting from cross wind, and also the mutual effects of the cooling towers in different arrangements have been presented.

The effects of wind on the flow pattern inside a single tower, and the formation of secondary, and vortical flows that are the cause of flow energy loss, can be seen in Figure $12 \mathrm{a}$.

In the type 1 arrangement the adverse effects of flow asymmetry resulting from the interference and interaction between the towers, and also, the secondary flows, and chocked regions due to wind are all present. In addition, the closeness of the two towers to each other causes an increase in the wind velocity between them; therefore, the pressure decreases in the vicinity of the radiators of this section, and the air inlet flow rate from these sections is 
H. Reshadatjoo et al.: Mechanics \& Industry 16, 602 (2015)

Table 4. Comparison of AIFRs for different tower arrangements under the effect of wind with a speed of 8 m.s ${ }^{-1}$.

\begin{tabular}{ccc}
\hline Arrangement type & $\begin{array}{c}\text { AIFR for towers on the } \\
\text { first line }\left(\mathrm{kg} . \mathrm{s}^{-1}\right)\end{array}$ & $\begin{array}{c}\text { AIFR for towers on the } \\
\text { second line }\left(\mathrm{kg} . \mathrm{s}^{-1}\right)\end{array}$ \\
\hline single tower (free convection) & 24723 & - \\
single tower (windy condition, 8 m.s $\left.{ }^{-1}\right)$ & 8765 & - \\
1 & 7580 & 11353 \\
2 & 8442 & 10850 \\
3 & 7842 & 11053 \\
4 & 7125 & - \\
\hline
\end{tabular}

reduced. Thus, in this type of arrangement, both factors responsible for limiting the amount of AIFR in towers are effective and cause a drastic reduction of AIFR.

The velocity vectors are shown in Figure $12 \mathrm{~b}$ on the central cross sections of towers, parallel to the wind direction.

With regard to Figure 12c, in the type 2 arrangement, where two cooling towers are positioned consecutively in a row, the conditions for the front line tower are similar to the type 1 towers; with the difference that, the factor responsible for velocity increase in the side radiators, which was caused by the closeness of the two towers, is eliminated in this case. Therefore, more flow rate passes through the tower on the first line, compared to the similar tower in the type 1 arrangement. Furthermore, the vortical region inside the second tower is smaller, relative to the front line tower which is directly exposed to the wind. This shows that the presence of the first tower in front relatively reduces the negative effects of the wind for the second tower. So, as is also shown in Table 4, the tower which is situated on the second line has more air intake flow rate, compared to the first tower. In the type 2 arrangement, the AIFR is almost $60 \%$ more, relative to the type 1 . Therefore, it can be stated, generally, that in the case of two cooling towers, the type 2 arrangement will have a better performance and efficiency than the type 1 arrangement.

In the type 3 tower arrangement, the two towers that are positioned on a line and in front are under dual effects of tower interactions and also direct impact of the wind (similar to the type 1 towers). In addition, the proximity of the two towers and the wind speed increase between them is a factor that has a direct bearing on their performance. But, since their flow rate is higher than that of the similar towers of type 1 , it may be reasoned that the tower which is located in the back, has had a positive influence on the performance of the front line towers. In addition, the third tower which is located on the second line, is affected less by the wind, due to the presence of the two towers in front; therefore, its air intake flow rate is more than that of the first line towers, Figure 12d.

In the type 4 arrangement, a combination of the phenomena existing in the type 1 and type 2 arrangements can be witnessed. These factors affect the AIFR for the first-line and second-line towers, as outlined in Table 4. It can be observed that the AIFR of the towers on the first line has been reduced, compared to the type 1 and type 2 tower arrangements. While, the AIFR of the second- line towers is less than that of the towers in the type 2 arrangement, and more than that in the type 1 .

\section{Conclusion}

In the present article, the air intake flow rate (AIFR) of Heller type dry natural cooling towers in different arrangements, in the conditions of free convection and wind gust were numerically investigated. With respect to the obtained results, the following conclusions could be made:

- In a state of free convection, factors such as the interference and interplay of plumes issuing from the towers, and the lack of symmetry due to tower interactions cause a reduction in the AIFR.

- By increasing the quantity of the cooling towers, the ill effects resulting from tower interactions intensify, and the AIFR is reduced.

- Wind, through four mechanisms, influences the flow pattern inside the tower and causes the reduction of the AIFR: (1) the increase of flow velocity around the side radiators, and the reduction of pressure difference between the inside and outside of the tower in these regions; (2) the flow deflection and deviation resulting from the wind flow running into the exiting plume, and the reduction of the effective cross section of the tower mouth; (3) the infiltration of the wind current inside the tower through the tower mouth, and the chocking of the flow inside the tower; and (4) the creation of secondary flows and vortical structures inside the tower.

- With the increase of the wind speed, the flow instability and its negative effects on the AIFR increase.

- Because the wind strongly influences the tower flow pattern, it has more adverse effects compared to the interactional effects of towers that were observed in the case of free convection.

- The AIFR is considerably more for the two towers which are situated consecutively and along the wind direction than the towers which are arranged in parallel and perpendicular to the wind direction.

- The arrangement of towers in parallel formation and perpendicular to the wind direction causes the flow velocity between the towers to increase, and the pressure to drop in this region, which disrupts the functioning of the radiators in this section. 
In different tower arrangements, the towers that are in the back row have a higher efficiency relative to the front row towers, because the adverse effects of the wind are endured and softened by the towers in front.

\section{References}

[1] D. Radosavljevic, D.B. Spalding, Simultaneous prediction of internal and external aerodynamic and thermal flow field of a natural draft cooling tower in a cross wind, Proc. 6th IAHR Cooling Tower Workshop, vol Pisa, 1988

[2] D.J. Bergstrom, D. Derksen, K.S. Rezkallah, Numerical study of wind flow over a cooling tower, J. Wind Eng. Ind. Aerodyna. 47 (1993) 657-664

[3] A.F.D. Preez, D.G. Kroger, Effect of wind on performance of a dry-cooling tower, Heat Recover. Syst. CHP 13 (1993) 139-146

[4] Q.D. Wei, B.Y. Zhang, K.Q. Liu, X.D. Du, X.Z. Meng, A study of the unfavorable effects of wind on the cooling efficiency of dry cooling towers, J. Wind Eng. Ind. Aerodyn. 54 (1995) 633-643

[5] D.D. Derksen, T.J. Bender, D.J. Bergstrom, K.S. Rezkallah, A study on the effects of wind on the air intake flow rate of a cooling tower: Part 1. Wind tunnel study, J. Wind Eng. Ind. Aerodyn. 64 (1996) 47-59

[6] M.D. Su, G.F. Tang, S. Fu, Numerical simulation of fluid flow and thermal performance of a dry-cooling tower under cross wind condition, J. Wind Eng. Ind. Aerodyn. 79 (1999) 289-306

[7] R.B. Bornoff, M.R. Mokhtarzadeh-Dehghan, A numerical study of interacting buoyant cooling-tower plumes, Atmospher. Environ. 35 (2001) 589-598
[8] N. Kapas, Investigation of flow characteristics of Hellertype cooling towers with different cooling delta angles, Periodica Politechnica ser. Mech. Eng. 2 (2003) 143-150

[9] R. Al-Waked, M. Behnia, The performance of natural draft dry cooling towers under crosswind: CFD study, Int. J. Energy Res. 28 (2004) 147-161

[10] M.R. Mokhtarzadeh-Dehghan, C.S. Konig, A.G. Robins, Numerical study of single and two interacting turbulent plumes in atmospheric cross flow, Atmospher. Environ. 40 (2006) 3909-3923

[11] Z. Zhai, S. Fu, Improving cooling efficiency of dry-cooling towers under cross-wind conditions by using wind-break methods, Appl. Thermal Eng. 26 (2006) 1008-1017

[12] N. Williamsona, S. Armfielda, M. Behnia, Numerical simulation of flow in a natural draft wet cooling tower - The effect of radial thermofluid fields, Appl. Thermal Eng. 28 (2008) 178-189

[13] H.G. Weller, G. Tabor, H. Jasak, C . Fureby, A Tensorial Approach to Computational Continuum Mechanics using Object-Oriented Techniques, Comput. Phys. 12 (1998) 620-631

[14] B.E. Launder, D.B. Spalding, The numerical computation of turbulent flows, Comput. Methods Appl. Mech. Eng. 3 (1974) 269-289

[15] H. Jasak, H.G. Weller, A.D. Gosman, High resolution NVD differencing scheme for arbitrarily unstructured meshes, Int. J. Numer. Methods Fluids 31 (1999) 431449

[16] A.O. Demuren, W. Rodi, Three-dimensional numerical calculations of flow and plume spreading past cooling towers, J. Heat Transfer 109 (1987) 113-119 